\title{
环青海湖斑头雁种群数量动态模拟及趋势分析
}

\author{
张耀南 ${ }^{1}$, 郝美玉 ${ }^{1}$ ，雷富民 ${ }^{2}$, 星 智 $^{3}$, 侯元生 ${ }^{3}$, 罗 泽 ${ }^{4}$ \\ (1. 中国科学院寒区早区环境与工程研究所, 甘肃 兰州 730000; 2. 中国科学院动物研究所, 北京 100101 ; \\ 3. 青海湖国家级自然保护区管理局, 青海 西宁 810000; 4. 中国科学院计算机网络信息中心, 北京 100190)
}

\begin{abstract}
摘要: 斑头雁是青海湖候鸟中的优势种之一, 其未来种群数量的变化对青海湖的保护决策具有重要作用。利 用 STELLA 图形化建模软件, 从动力学出发, 构建了青海湖地区斑头雁种群数量变化的动态模型。在该模型中, 根据前人和青海湖自然保护区对斑头雁的观测研究结果, 设置了班头雁的交配、产卵、孵卵、育幼、迁入、迁出、 死亡等过程的模型参数。根据斑头雁在青海湖的活动时间, 设置以年为模拟单位, 选取 $1 / 7$ 为模拟步长, 对未来 25 年的青海湖斑头雁种群变化趋势进行了模拟, 并进行了灾害模拟。模拟结果表明, 未来青海湖斑头雁的最大种 群数量, 将在 20000 余只的饱和数量之内变化。2006-2008 年斑头雁观测数据验证表明, 该模型模拟结果可信, 其方法对青海湖的其他鸟类研究也具有一定的参考价值。
\end{abstract}

关键词: 斑头雁; 青海湖; 种群数量; 动态模型

中图分类号: Q959.7; Q958.115 文献标识码: A 文章编号: 0254-5853-(2009)05-0578-07

\section{Simulation of Population Dynamics of Bar-headed Geese (Anser indicus) Around Qinghai Lake Region and Trend Analysis}

\author{
ZHANG Yao-nan ${ }^{1}$, HAO Mei-yu ${ }^{1}$, LEI Fu-ming ${ }^{2}$, XING Zhi $^{3}$, HOU Yuan-sheng ${ }^{3}$, LUO Ze ${ }^{4}$ \\ (1. Cold and Arid Regions Environmental and Engineering Research Institute, CAS, Lanzhou 730000, China; \\ 2. The Institute of Zoology, CAS, Beijing 100101, China; 3.Qinghai Lake National Nature Reserve, Xining 810000, China; \\ 4. Computer Network Information Center, CAS, Beijing 100190, China)
}

\begin{abstract}
Bar-headed geese (Anser indicus) is animportant migratory goose species group in the Qinghai lake region. Population dynamics of the group provides the foundation data of decision-making in Bar-headed geese protection in Qinghai lake National Nature Reserve. A bar-headed goose population dynamics model was constructed in Qinghai lake region regarding the dynamics with STELLA graphical modeling software. Parameters included in the model described mating, egg deposition, hatching, brood-rearing, immigration, emigration and mortality based on long term observation data. In running simulation models, we use one year as unit and 1/7 as a time step in accordance of the periodic time bar-headed geese stay in Qinghai lake. The next 25 years simulated population change results showed that the trend of the maximum bar-headed goose population will vary around 20 thousand in Qinghai lake region. Meanwhile, the population was simulated assuming calamity strikes in 2010. The model and simulated results of the bar-headed goose population are reliable according to calibrated results using observed data from 2006 to 2008, and the method of modeling bar-headed goose population provides some reference to further research on other birds in Qinghai lake region. Finally, suggestions of protection for the bar-headed goose have been proposed based on above results.
\end{abstract}

Key words: Bar-headed goose; Qinghai lake area; Populations; Simulation model

斑头雁活动于高寒地带, 青海湖是其重要的繁 殖栖息地之一。青海湖斑头雁的交配、营巢、产卵、 孵卵、育雏、换羽等繁殖习性已有一定的观察记录 (Wang, 1981; Xian, 1964)。繁殖后的斑头雁在
青藏高原，如西藏雅鲁藏布江、拉萨河、年楚河的 越冬行为以及种群数量和分布也有相应的研究

(Song et al，1994)。Herbert et al（2004）于 1998、 2000 和 2002 年 3 次对在印度 Ladakh 地区越冬的斑

收稿日期：2009-03-12；接受日期：2009-07-09

基金项目：国家基础科学人才培养科学基金（J0630966）；中国科学院信息化专项项目（INFO-115-D02）

第一作者简介: 张耀南(1966-), 男, 甘肃省秦安人, 中国科学院寒区早区环境与工程研究所研究员, 主要从事数据、模型、计算与 e-science 研究。E-mail:yaonan@1zb.ac.cn 
头雁的种群数量、种群结构等方面也做了详细的观 察记录和研究, 区分了斑头雁栖息的 7 种湿地生 境, 定量分析了各生境中斑头雁的家族大小及幼雁 比例, 估计了幼雁的死亡率为 $1.4 \% / \mathrm{d}$ 。此研究区也 为高寒区, 且观测的数据具有代表性, 为青海湖地 区斑头雁的研究奠定了基础。斑头雁未来在青海湖 的种群动态变化, 对于青海湖自然保护区的决策制 定有重要的参考作用, 但关于斑头雁种群数量的动 态变化研究国内外都不多见。国外有一些关于鸟类 的研究模型(Letcher et al, 1998; Liu, 1993; Liu et al, 1995; Mason et al, 1996), 但利用动力学原理建立模 型来研究种群数量变化多数集中在害虫( Zhu et al, 1994; Cheng, 1995; Wen et al, 1994; Zhou \& Lou, 1992; Guo et al, 1993; Wu et al,1990; Chen \& Li, 1993; Zhang \& Wang, 1990)、昆虫(Ju \& Shen, 2005) 和鼠类(Zhang et al, 1990)的研究中, 而研究鸟类的 很少。本文尝试利用 STELLA(Isee Systems, 2006) 这一动力学建模软件, 从动力学角度对青海湖斑头 雁从开始繁殖到越冬期之前的种群动态变化情况 建立模型, 结合青海湖自然保护区多年来在环青海 湖区的观察记录和我们的实际考察, 参考前人关于
斑头雁研究文献的一些参数 (Wang, 1981; Herbert et al, 2004; Gao, 1988; Zheng \& He, 2006), 利用建立 的动态模型, 对该地区的斑头雁种群数量动态变化 情况进行模拟与变化趋势的初步预测分析。

\section{1 研究区概况}

青海湖是中国最大的内陆咸水湖, 湖面海拔 $3100 \mathrm{~m}$, 是中国西部重要的候鸟栖息地。环湖区是 国家级自然保护区, 地处北纬 $36^{\circ} 30^{\prime} \sim 37^{\circ} 20^{\prime}$, 东经 $99^{\circ} 30^{\prime} \sim 100^{\circ} 50^{\prime}$, 面积约 $4952 \mathrm{~km}^{2}$, 主 要保护高原湿地生态系统和各种珍稀鸟类。斑头雁 在青海湖的活动范围集中在保护区之内, 研究区域 仅限于保护区(图 1)。斑头雁迁徙路线相对较固定

(Huang \& Zhang, 2007)，3 月份从越冬地陆续迁徙 至青海湖, 先在各湿地分散栖息, 补充在飞行过程 中消耗的体能, 然后集中到繁殖地繁殖。整个过程 中也伴有不参加繁殖的斑头雁的迁入和迁出活动。

9 月份返回到越冬地。斑头雁是以食草为主的水禽, 一般分布在环湖的河口湿地和沼泽区栖息。斑头雁 在青海湖环湖区的分布也较为固定, 繁殖地和栖息 地一般是分开的。主要繁殖地集中在蛋岛和三块石

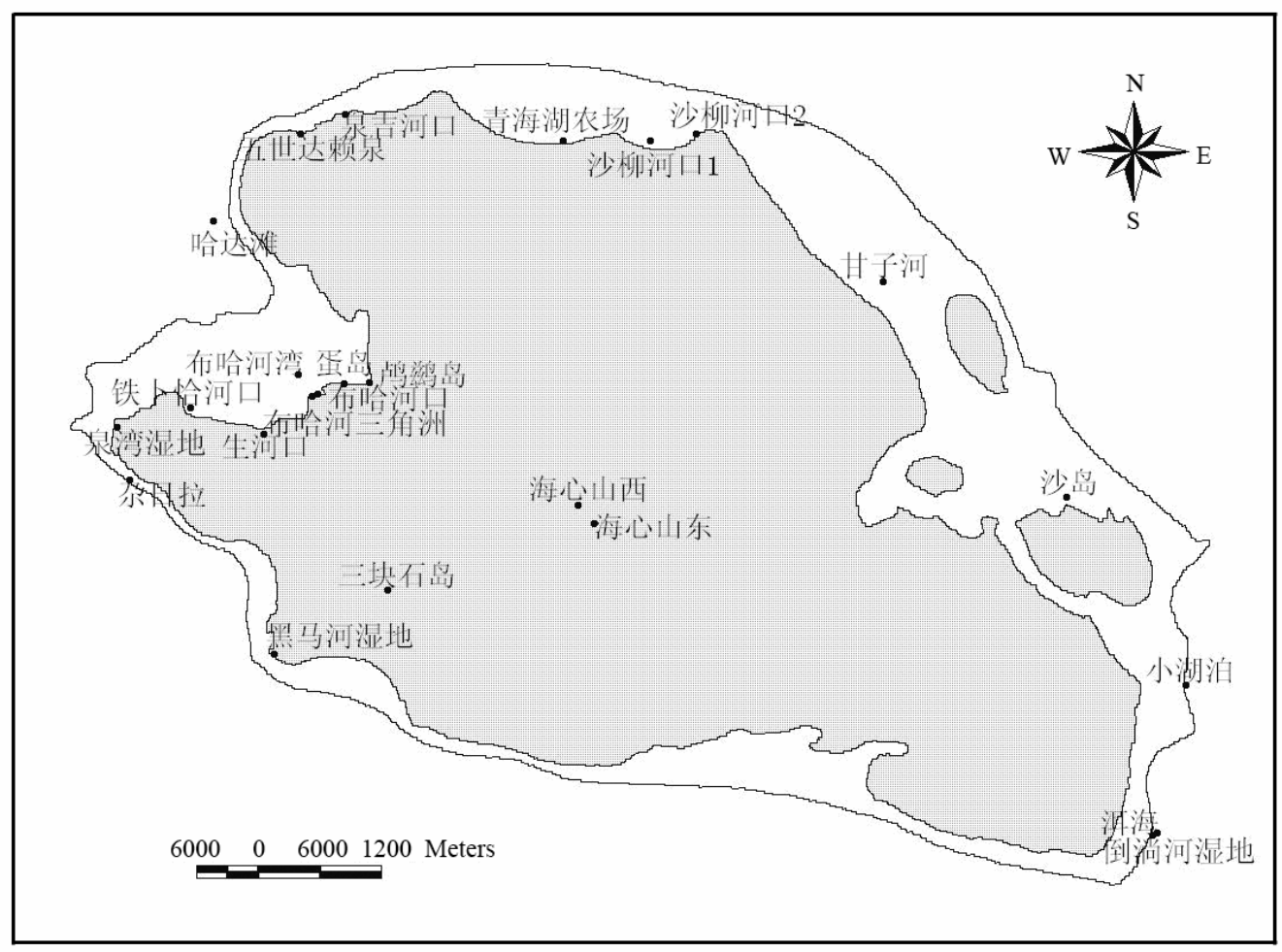

图 1 斑头雁在环青海湖地区的分布图

Fig.1 The distribution of bar-headed goose around Qinghai lake area 
岛。繁殖结束后, 成雁会带着自己的幼雁迁徙到安 全隐蔽的环湖湿地栖息、受食, 并完成育幼。斑头 雁种群在环青海湖的西北方向 (主要集中在布哈河 和泉湾河口湿地) 分布较集中, 东南方向分布较少。

\section{2 方 法}

\section{1 数据来源}

斑头雁是集群性的水禽, 飞翔能力很强, 活动 的范围较大, 数据获取困难。本模型中的一些输入 参数, 主要根据青海湖自然保护区斑头雁的观测数 据、实地考察数据、历年文献中记载以及从前人研 究的结果中获得, 具体参数的设置见下文。

\section{2 模型构建}

采用 Isee systems 公司开发的基于图标的 Stella 8.1 .1 建模软件, 按照斑头雁的生命过程, 构建斑头 雁的动态模型。模型共分 3 部分, 主要反映从幼雁 出生、成熟, 以及成雁死亡的整个过程。第一个部 分反映幼雁出生阶段。作为初始值输入模型的成雁 种群数量, 经过交配、产卵、孵卵后, 得到幼雁的
种群数量。这个过程受成年斑头雁次年返回青海湖 的系数、环境容纳量、交配率、产卵量和卵的孵化 率的制约。第二个部分反映幼雁成熟阶段。由模型 第一个部分得到的幼雁种群数量, 剔除死亡幼雁, 剩下的全部存活为成雁。这个过程主要受幼雁的死 亡率制约。第三个部分反映成雁死亡。存活的幼雁 都长成为成雁, 成雁种群伴随正常死亡和迁入迁出 迁徙活动, 并受环境生境因子的制约。本模型中采 用迁入和迁出模拟来表达生境因子对成年斑头雁 的影响。按照斑头雁的生命过程, 建立了包括成雁 交配、产卵、孵卵、育幼、迁入、迁出、死亡和幼 雁死亡等过程的动态模型, 并加入了发生灾害的模 块。模型以年为单位, 每年模拟一次, 模拟步长取 $1 / 7$ (模拟步长的选取与斑头雁每年在环青海湖区停 留的月份一致)。模型每年的模拟输出的种群数量 乘以一个比例系数作为模型下一年的输入。本文利 用建立的模型，从 2006 年开始，对未来 25 年的斑 头雁种群数量变化情况进行了模拟。

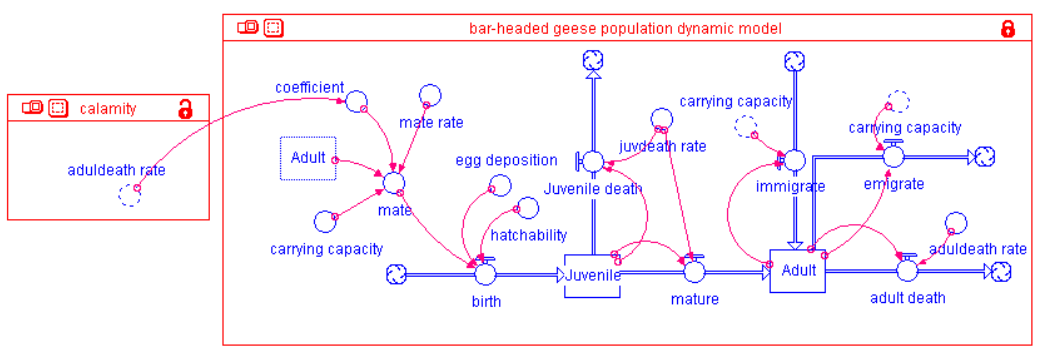

图 2 斑头雁种群数量动态变化模型

Fig.2 Population dynamics model of bar-headed goose

coefficient: 返回系数值; mate rate: 交配率; carrying capacity: 环境容纳量; mate: 交配; egg deposition: 孵卵量; hatchability: 卵的 琈化率; birth: 幼雁的出生; Juvenile: 幼雁的种群数量; Juvenile death: 幼雁的死亡; Juvdeath rate: 幼雁的死亡率; mature: 幼雁的成 熟; Adult: 成雁的种群数量; immigrate: 成雁的迁入; emigrate: 成雁的迁出; adult death: 成雁的死亡; aduldeath rate: 成雁的死亡率; calamity: 灾害模块。

\section{3 参数估计}

2.3.1 返回系数值 在不清楚影响斑头雁次年返 回青海湖数量的背景下, 根据青海湖自然保护区工 作人员多年的观察和分析结果, 采用一个比例系数 来确定成年雁的模型初始输入值, 并将返回系数在 模型中设计为可调节量, 模型中初始值以前一年斑 头雁总数为基数乘以返回系数确定。由于 3-4 月 份斑头雁陆续返回时, 湖面还未完全溶化, 三块石 岛和海心山岛上的数据无法测得, 2008 年 3 月底, 尔日拉、黑马河、哈达滩、泉湾湿地和江西沟共有 斑头雁 3302 只, 2007 年 8 月初, 黑马河、泉湾湿 地、蛋岛、布哈河口、铁卜恰河口、沙柳河口、泉
吉河口、尔日拉、沙岛、哈达滩和深河口共有斑头 雁 4592 只，斑头雁返回系数值为 $73 \%$ 。由于斑头 雁在这两个时间段分布较分散, 部分隐蔽地方缺 测, 且一般分布数量较小，作为两个时间段的比值 误差较小。如果发生类似禽流感这样的灾害时, 斑 头雁的繁殖个体数与发生灾害之前相比较为接近

(Zhang et al, 2008), 所以在发生灾害时, 斑头雁 在繁殖后期的数量将下降, 次年的返回数量变化不 大，返回系数值将增大。本次灾害模拟时，假定灾 害发生后返回系数为 $75 \%$ 。

2.3.2 环境容纳量 自然状态下, 种群总是处于有 条件限制的环境当中变化。种群增长随着种群密度 
的上升, 种群内部对食物资源以及生境空间资源的 竞争也将加剧。这种竞争必然会影响到种群的出生 率和死亡率, 从而降低种群的实际增长率, 直至种 群停止增长, 甚至发生下降 (Qin et al, 2008)。斑 头雁和其他种群一样, 种群数量的增长受到了生境 等因素的制约, 符合有限环境种群增长的微分方 程: $\mathrm{d} N / \mathrm{d} t=r N(1-N / K)$, 其中 $r$ 为潜在增值能力, $N$ 为种群数量, $K$ 为环境容纳量, $t$ 为时间。青海湖 2004 年观测到的斑头雁的数量为 19700 只 (Zheng $\& \mathrm{He}, 2006)$, 这是从 1960 年有斑头雁记录(Xian, 1964) 以来种群数量比较多的记录。根据历年观测数 量和观测者的估计, 将环境容纳量的饱和值设置围 绕 22000 只上下波动。

2.3.3 交配 斑头雁 3 月份从越冬地返回到青海湖 后, 多数已经配成对进入交配, 还有部分是在青海 湖进行配对并进入交配。第一年出生的幼雁, 在次 年返回青海湖时不参与交配。交配后的成年斑头雁 失偶后成为孤雁, 也有一些成雁交配不成功。本模 型中引用 Gao (1988) 一文中孤雁的最大比例 $2 \%$, 认 为交配成功率 $98 \%$ 。

2.3.4 出生 每对成年斑头雁平均产卵为 4 6 个。 模型假定产卵数服从均值为 5 , 标准差为 0.1 的正 态分布。据 Wang (1981) 观察得到卵的孵化率约 为 $61 \%$, 未孵化出的大部分为垫底卵。在孵化的过 程中, 集群的中心地带斑头雁的孵化率较高, 边缘 地带的斑头雁的睬化率较低。

2.3.5 成熟 刚出生的幼雁抵抗外界的能力较弱, 死亡率较高。据 Xian et al (1964) 在 1960 年的观测 情况可知, 刚出生的幼雁在成年雁领着学游泳以及 从三块石游向河口湿地的过程中, 体弱的幼雁常常 被海浪淹没, 导致体弱幼雁的大量死亡。再加上鱼 鸥和鸬鹚等在幼雁刚出生时的袭击敌害, 大大降低 了幼雁的成活率。据 Herbert et al (2004)观测, 幼雁 在 7 月中旬为 700 只, 在 9 月初为 353 只, 死亡率 约 50\%。其余的幼雁, 模型认为全部存活并成长为
成年雁。禽流感的爆发, 一般在春季迁徙回来初期 比较常见。在本模型的灾害模拟中, 假定在繁殖前 期发生，不会影响幼雁的死亡率。

2.3.6 迁出和迁入 受环青海湖的湿地生境的限 制, 斑头雁的种群数量不可能无限制的增长。环境 条件决定了斑头雁迁出和迁入青海湖的动态过程, 在本模型中通过迁出和迁入参数来模拟湿地生态 系统的平衡因子。此处的迁入和迁出不是指 3 月和 9 月大批斑头雁的迁徙, 而是指在青海湖繁殖或换 羽期间, 受生境容纳量的制约, 斑头雁种群自行迁 入和迁出的调节过程。此处迁出系数为 0.1 , 迁入 系数为 0.05 , 对种群数量的变化影响较小, 作为一 个随机数。总的影响趋势是减小种群的数量, 针对 短期停留的斑头雁而言。

2.3.7 死亡 这里的死亡是指成年斑头雁的死亡。 成年斑头雁的死亡是环青海湖区和越冬地两地死 亡的总和。据有关研究文献介绍, 斑头雁在换羽期 间失去了飞行能力, 很容易受到敌害的袭击, 死亡 率相对较高。在飞往越冬地的过程中, 容易发生事 故而导致死亡。斑头雁的正常寿命一般为 12 15 年, 每年的正常死亡也占一定的比例。综合以上各方面 的因素, 成年斑头雁每年的死亡率估计为 $20 \%$ 。如 果发生类似禽流感的灾害时, 斑头雁的成雁死亡率 将会增大, 此处假定成雁死亡率为 50\%进行灾害情 况的模拟。

\section{3 结果与分析}

表 1 中模拟数据与实测的数据的对照, 发现模 拟数据与实测数据相差较小, 而且在 3 年之内斑头 雁种群数量的变化趋势是趋于增长的, 这与实测数 据的变化趋势是一致的。

实测数据来源于青海湖自然保护区。2006 年 6 月初的实测数量缺少蛋岛、三块石岛和海心山三个 重要地点的数据, 6 月底的数据缺测。

表 1 斑头雁种群数量的实测值与模拟值的比较

Tab. 1 Comparison between observed and simulated population of bar-headed goose

\begin{tabular}{lcccc}
\hline \multirow{2}{*}{ 年份 Year } & \multicolumn{2}{c}{ 实测数量 (只) } & Number of the observed & \multicolumn{2}{c}{ 模拟数量 (只) } & Number of the simulated \\
\cline { 2 - 5 } & 6月初 Early June & 6月底 Late June & 最小值 Minimum & 峰值 Maximum \\
\hline 2006 & 3096 & - & 3598 & 5249 \\
2007 & 6721 & 4929 & 5439 & 6413 \\
2008 & 6595 & 7641 & 6545 & 7355 \\
\hline
\end{tabular}




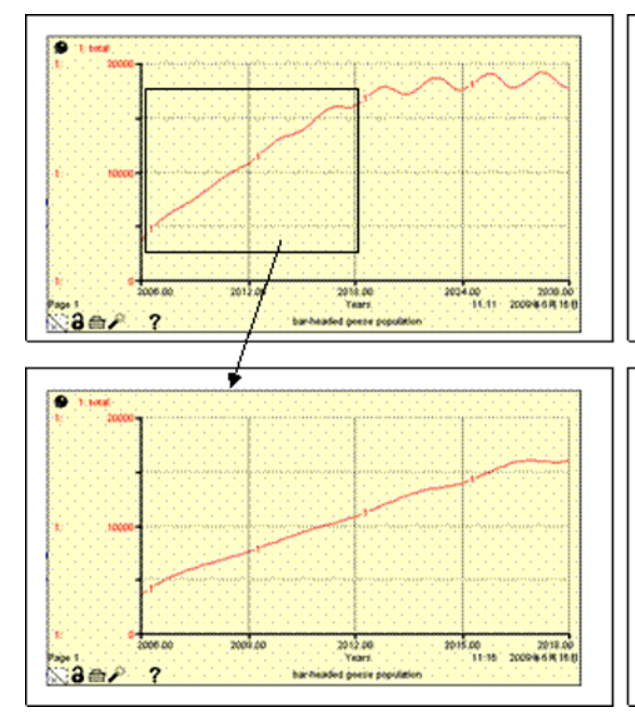

a

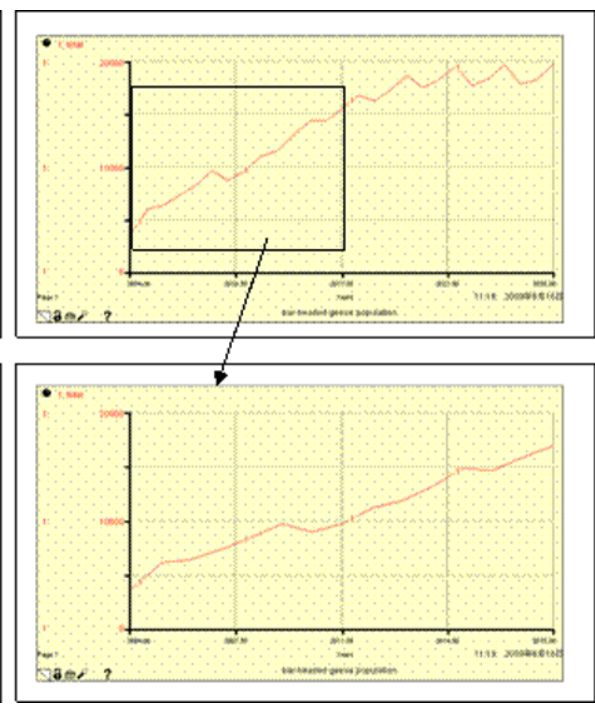

$\mathrm{b}$

图 $32006-2030$ 年的斑头雁种群数量变化模拟结果

Fig. 3 Simulation results of population dynamics of bar-headed goose in 2006-2030

a: 环境条件相对稳定状态的模拟结果； b: 假定 2010 年发生灾害的模拟结果。

$\mathrm{a}$ is the simulated result of a relatively stable state; $\mathrm{b}$ is the simulated result that assuming calamity strikes in 2010 .

通过模拟发现, 成年斑头雁的初始值影响曲线 的斜率, 即影响到达饱和状态的时间。返回系数值 的大小、成年斑头雁和幼雁的死亡率, 影响达到饱 和后的种群数量。幼雁的死亡率较成年斑头雁死亡 率的影响小一些。根据历年繁殖期观测到的斑头雁 数据以及 2006 年 3 月份的部分实测数据, 再加上 未观测区域 (海心山和三块石岛) 的经验值, 在模 型中经过权衡, 取成年斑头雁的初始值为 3598 只。 在模拟中, 当返回系数值取 0.73 , 幼雁的死亡率为 0.5 , 成年斑头雁的死亡率为 0.2 时, 2006 年到 2030 年的斑头雁种群数量模拟结果如图 3a 所示。假定 2010 年发生类似而流感的灾害时, 模拟结果如图 $3 b$ 所示。2005 年在青海湖保护区内的野生鸟发生 了严重的禽流感病毒的感染（Zheng \& He, 2006), 斑头雁的死亡数量较大, 再加上 2005 年以前的数 据不详, 因此 2005 年以前斑头雁种群数量没有引 入模型进行模拟, 但在模拟中进行了参照。从 2006 年开始对 2006-2008 年的种群数量进行了模拟, 并对未来 22 年的斑头雁的种群数量变化趋势进行 了初步预测分析。由模拟可知, 从 2006 年开始, 斑头雁种群数量一直处于增长状态, 到 2012 年增 长趋于缓和, 增长幅度变小, 2018 年后基本趋于稳 定, 达到 2 万余只。如果发生类似禽流感的灾害时, 斑头雁在青海湖的繁殖个体数量变化不大。

\section{4 讨 论}

\section{1 模型特征}

模型假定情景是, 斑头雁群居的青海湖环境条 件相对稳定, 没有自然灾害和大型传染病毒的影 响, 只考虑了食物和生境条件等对其种群数量的影 响。模型从相对稳定环境情景出发, 对环青海湖湿 地区的斑头雁种群数量的时间动态变化进了模拟, 模拟结果是可信的。斑头雁的种群数量在该地区的 动态模拟结果, 可为科学合理的保护和管理决策提 供了依据。

模型还假定发生类似禽流感的灾害时, 斑头雁 在青海湖的增长的趋势基本不变, 最终因受环境条 件的限制斑头雁种群数量仍会达到一个稳定状态, 达到稳定状态的时间也相差不大。如果考虑迁徙中 短时间停留青海湖的斑头雁, 斑头雁的总数有可能 超过稳定状态的饱和值。

在数据缺乏的情况下, 本模型中一些参数很难 确定, 为了使模型可行, 将对斑头雁的成雁的死亡 率进行了估计, 以便使用者根据研究的需要和具体 应用的情况, 通过调节变量来调整模型, 将该模型 运用于其他地区的斑头赝研究或其他有同样繁殖 习性的鸟类的研究。

模型模拟过程与一年内实际种群数量变化过 
程是有区别的。模型中模拟过程是以年为单位, 模 拟步长为 $1 / 7$, 斑头雁每年在环青海湖停留时间约 为 7 个月。而在实际的繁殖地, 斑头雁种群数量经 历先增长至达到高峰期, 后逐渐减少的过程。由于 每年每只幼雁的出壳期不同, 成年斑头雁迁出繁殖 地的时间也不同, 所以, 达到繁殖高峰期的时间在 不同的年份会有不同。繁殖结束后, 分散到隐蔽的 湿地进行育幼, 也是种群数量减少的过程。

本模型存在一些不足, 尚待进一步完善。如模 型中没有引入环青海湖植被、气候、湿地大小等影 响因子对斑头雁种群的影响, 没有将斑头雁在越冬 地的种群数量动态变化情况引入模型。其次, 斑头 雁在青海湖不是单一存在的物种, 是与其他的鸟类, 如鸬兹 (Phalacrocorax carbo)、棕头鸥 (Larus brunnicephalus) 等共存的, 虽然对食草的斑头雁在 食物资源的生境上竞争不明显, 但在环青海湖地区 鸟类繁殖地生境空间上还是存在明显的竞争。随着 气候变化, 环青海湖湿地的面积也在发生变化, 鸟 类之间的竞争会变得强烈。因此, 青海湖鸟类之间 的竞争的因素也应该是斑头雁种群变化模型考虑 的影响因子。

\section{2 保护与管理建议}

斑头雁属于国家珍稀保护的动物, 分布于中国 西北、东北、华北、长江流域以南, 以及亚洲中部、 克什米尔、蒙古、印度、巴基斯坦和缅甸等地。斑 头雁在我国分布尚有一定数量 (具体种群数量不 详), 但也面临威胁。斑头雁被列入《国家保护的

\section{参考文献:}

Chen XF, Li DM, 1994. A study on the population dynamics simulation of Dendrolius punctatus[J].Sci. Silv. Sin, 36(1): 88-92.[陈晓峰, 李典谟. 1994. 马尾松毛虫种群动态及模拟研究. 林业科学, 30(1): 88-92.]

Cheng MK. 1995. Studies on population number dynamic simulation of tea green leafhopper during its first population peak period in the years of the double population peaks [J]. J Anhui Agric Sci, 23(4): 340-341.[程 鸣珂. 1995. 茶小绿叶蝉一峰期种群数量的动态模拟研究. 安徽农 业科学, 23(4): 340-341.]

Cornwell L, Costanza R. 1994. An experimental analysis of the effectiveness of an environmental assurance bonding system on player behavior in a simulated firm[J]. Ecol Econ, 11: 213-226.

Gao YH. 1988. Primary exploration of several ecological problem of bar-headed geese in Egg Island [J]. Chn J Wildl, 42(2): 21-22. [高元洪. 1988. 初探岛岛斑头雁几个生态问题. 野生动物, 42(2): 21-22.]

Guo JX, Li JC, Zhao WC, 1993. Aphid population dynamic and long-term prediction in the wheat winter field in Heibei province [J]. Acta Agric Boreall-Sin, 8(2): 16-20. [郭金霞, 李建成, 赵文臣. 1993. 河北省穗
有益的或者有重要经济、科学研究价值的陆生野生 动物名录》。随着适合斑头雁生存的湿地生境的减 少, 斑头雁的分布和活动范围较为集中。据跟踪调 查, 分布在西北的斑头雁, 越冬和繁殖基本集中在 青藏高原地区。为了使斑头雁能够在青海湖保护区 内永续的繁殖和发展, 建议从以下几个方面加强研 究。

（1）栖息地和繁殖地的保护是一项长期和艰 巨的任务。尤其是繁殖地的保护。繁殖地的位置在 青海湖地区基本固定，三块石岛坐落在湖体的中 间, 面积受青海湖水位的涨落等自然因素影响较 大, 受人为干扰较小。蛋岛的面积则受自然和人为 的影响都较大。因此, 加强繁殖地保护工作是至关 重要的。繁殖后的斑头雁主要依赖与环湖湿地生 境, 因此尽可能保护或适当增大湿地生境的面积, 对增大环境容纳量非常重要。保护同时, 需要加强 湿地生境与各自然因子的相互关系的研究, 使保护 工作落到实处。

（2）加强未孵化卵的人工孵化力度。斑头雁 在繁殖地未孵化的卵约占 $39 \%$, 占总产卵数的比例 较大; 人工孵化雁的回收率也较野生的高 (Gao, 1988)。所以, 对未孵化卵的有效利用是非常重要的。

（3）保护和扩大斑头雁的迁徙通道。适合斑 头雁生存的湿地生境的减少, 使其迁徙的通道越来 越少, 分布也变得越来越集中。扩大斑头雁的迁徙 通道, 使其分布变得分散, 寻求更多可利用的生境, 将对斑头雁种群数量的增长有一定的影响作用。
期麦蚜预测及种群动态模拟. 华北农学报, 8(2): 16-20.]

Herbert HT, Prins, Sipke E van W. 2004. Number, population structure and habitat use of bar-headed geese Anser indicus in Ladakh (India) during the brood-rearing period[J]. Acta Zool Sin , 50(5): 738-744.

Huang Q, Zhang LH. 2007. New record of birds in ChongQing [J]. Sichuan J Zool, 26(3): 594. [黄 强, 张罗虹. 2007. 重庆市鸟类新记录一 斑头雁. 四川动物, 26(3): 594.]

Isee Systems. 2006. Product info of STELLA [EB/OL]. (http://www.iseesystems.com).

Ju RH, Shen ZR. 2005. Review on insect population dynamics simulation models[J]. Acta Ecol Sin, 25(10): 2710-2716. [句荣辉, 沈佐锐. 2005. 昆虫种群动态模拟模型. 生态学报, 25(10): 2710-2716.]

Letcher BH, Priddy JA, Walters JR. 1998. An individual-based, spatially-explicit simulation model of the population dynamics of the endangered red-cockaded woodpecker Picoides borealis[J]. Biol Conser 86: 1-14.

Liu JG. 1993. ECOLECON: An ecological-economic model for species 
conservation in complex forest landscapes[J]. Ecol Mod, 70: 63-87.

Liu JG, Dunning JB Jr, Pulliam HR. 1995. Potential effects of a forest management plan on Bachman' s Sparrows (Aimophila aestivalis): Linking a spatially explicit model with GIS[J]. Conser Biol, 9: 62-75.

Mason DM, Brandt SB. 1996. Effects of spatial scale and foraging efficiency on the predictions made by spatially-explicit models of fish growth rate potential[J]. Environ Biol Fish 45: 283-298.

Qin Z, Zhang JE, Luo SM. 2008.Application of system dynamic modeling software in the population ecology[J]. J Anhui Agr.ic Sci, 36(26): 11615-11617. [秦 钟, 章家恩, 骆世明. 2008. 系统动力学模拟软件 在种群生态学中的应用. 安徽农业科学, 36(26): 11615-11617.]

Song YL, Canjue ZM. 1994. Bishop MA. Bar-headed geese Anser indicus wintering population number and distribution in south-central Tibet Yarlung Zangbo River region[J]. Chn J Zool, 9(2): 27-30. [宋延龄, 仓 曲卓玛. 1994. Mary AB. 西藏雅鲁藏布江中游地区斑头雁越冬种群 数量和分布. 动物学杂志, 29(2): 27-30.]

Tao ZP, Li MY. 2007. What is the limit of Chinese coal supplies - A STELLA model of Hubbert Peak[J]. Energy Policy, 35: 3145-3154.

Wang X. 1981. Observation of bar-headed geese breeding behavior[J]. Chn $J$ Wildl, (3): 29-31. [王侠. 斑头雁繁殖习性的观察. 野生动物, (3): 29-31.]

Wen XJ, Sun SX, Li XJ. 1994. Studies on the population dynamics of Dendrolimus spectabilis butler [J]. Sci Silvae Sin, 30(1): 39-43. [温秀军, 孙士学, 李向军. 1994. 赤松毛虫种群数量动态的研究. 林业科学, 30(1): 39-43.]

Wu ZF, Yan YJ, Zhao SX ,1990. Studies on the population dynamic simulation of brown planthopper[J]. J Fujian Agric Coll,e 19(2): 115-122. [吴中孚, 严英俊, 赵士熙. 1990. 褐稻䖝田问种群动态模拟 的研究. 福建农学院学报, 19(2): 115-122.]

Xian YH. 1964. Preliminary observation of bar-headed geese breeding behavior in Qinghai lake area[J]. Chn J Zool, 6(1): 12-14.[冼耀华.
1964. 青海湖地区斑头雁繁殖习性的初步观察. 动物学杂志, 6(1): 12-14.]

Ying OY. 2008. Modeling the mechanisms for uptake and translocation of dioxane in a soil-plant ecosystem with STELLA[J]. J Contam Hydrol, 95: $17-29$.

Ying OY, Huang CH, Huang DY. 2007. Simulating uptake and transport of TNT by plants using STELLA[J]. Chemosphere, 69: 1245-1252.

Zhang GG, Liu DP, Jiang HX. 2008. The current status of waterbirds after avian influenza outbreak at Qinghai Lake, China [J]. Chn J Zool, 43(2): 51-56. [张国钢, 刘东平, 江红星. 2008. 㥐流感发生后青海湖水鸟的 种群现状. 动物学杂志, 43(2): 51-56.]

Zhang ZB, Zhu J, Yang HF. 1990. Modelling of seasonal dynamics of Cricetulus triton population in Heibei, China[J]. Acta Zool Sin, 36(2): 136-143. [张知涁, 朱 靖, 杨荷芳. 1990. 大仓鼠种群季节动态的模 拟模型. 动物学报, 36(2): 136-143.]

Zhang WJ, Wang SZ, 1990. A simulation model of cotton Aphid population [J]. $J$ Ecol,, 9(5): 26-28. [张文军, 汪世泽. 1990. 棉蚜种群数量模拟 模型的初步研究. 生态学杂志, 9(5): 26-28.]

Zheng J, He YB. 2006. Thinking of wild birds avian flu protection and control in Qinghai lake area [J]. Sci Technol Qinghai,(1)：8-9.[郑 杰, 何玉邦. 2006. 对青海湖区野生鸟类禽流感疫情防控的思考. 青海 科技, (1): 8-9.]

Zhou JZ, Luo ZY. 1992. The simulation models for the population dynamics of citrus red mite [J]. Acta Ecol Sin, 12(4): 348-355. [周建中, 罗志义. 1992. 柑桔全爪螨自然种群动态的模拟模型. 生态学报, 12(4): 348-355.]

Zhu ZR, Cheng JA, Huang CW. 1994. Simulation analysis of white-backed planthopper population dynamics on first season rice in ZheJiang province, China[J]. Acta Ecol Sin, 14(2): 188-195.[祝增荣, 程家安, 黄次伟. 1994. 白背飞䖝种群动态的模拟研究. 生态学报, 14(2): 188-195.]

\section{“环青海湖斑头雁种群数量动态模拟及趋势分析”项目组简介}

研究方向：主要从事数据、模型和高性能计算应用及 e-science 研究, 致力于数据驱动的模型研究和 模型的综合集成。

队伍情况: 目前共有 20 人的研究队伍。有研究员 2 人，高级工程师 1 人，助研和工程师 5 人。在读 博士生 4 人、硕士研究生 5 人, 兼职教授 1 人。

承担的项目: 目前主要开展中国科学院信息化专项支持黑河流域生态环境集成研究的 e-science 项目、 青海湖区域重要野生鸟类资源及疫病监测与风险评估研究 e-Science 应用项目。开展黑河流域交叉综合集 成空间建模与模拟环境项目，分布式水热耦合模型研究。还承担国家自然科学基金委员会特殊学科人才培 养基金冰川学冻土学特殊学科点项目。承担国家基础条件建设项目特殊环境与灾害研究网络数据平台建设 项目等 12 个项目。

主要成果：通过数据资源体系、模型资源库与高性能计算的结合, 构建了 3M（监测平台、模型平台、 计算操作平台) 平台, 形成模型开发、模型集成、模型模拟的综合集成空间环境和资源环境与生态研究的 e-science 基础设施, 并利用该环境开展了一些针对环境与生态的示范性的模型建立和应用研究。

张耀南 